An unregistered TARDBP mutation in a case presenting with young-onset dementia

\author{
Mahmoud Gad ${ }^{1 \star}$, Walid Nasr ${ }^{1}$ and Tareq Qassem ${ }^{2}$ \\ ${ }^{1}$ Al-Amal Hospital, Ministry of Health and Prevention and \\ ${ }^{2}$ Maudsley Health, Al-Amal Hospital, Ministry of Health and \\ Prevention, Mohammed Bin Rashid University Of Medicine and \\ Health Sciences, Institute of Psychiatry, Ain Shams University \\ ${ }^{*}$ Corresponding author.
}

doi: 10.1192/bjo.2021.338

Objective. This poster aims to report an unregistered mutation in Transactive Response DNA Binding Protein (TARDBP) gene in a patient presenting young-onset dementia.

Hypothesis: Novel heterozygous mutation in the TARDBP gene is linked to a case of with young-onset dementia.

Background. Pathogenic variants in TARDBP cause autosomal dominant fronto-temporal degeneration, characterized by TDP43-positive inclusions, dystonia, dyslexia, receptive dysphasia, and paraphrasic errors. In addition to the neurocognitive deficits, patients might suffer from cardiomyopathy and amyotrophic lateral sclerosis.

Case report. Molecular genetic analysis of whole-exome sequencing (WES) was carried out for a 45 -year-old male patient presenting with cognitive decline and behavioural symptoms.

Discussion. WES Identified the heterozygous variant c.527A $>\mathrm{T}$ p.(Lys 176lle) in TARDBP gene. To the best of our knowledge the variant has not been described in the literature so far (HGMD 2019.3). No allele frequencies in the general population have been documented.

Conclusion. We believe that we have identified a novel mutation in the TARDBP gene. This mutation is likely to be linked to this patient presenting with young-onset dementia.

\section{Off-licence use of clozapine in patients with emotionally unstable personality disorder: a case series analysis

\author{
Declan Hyland ${ }^{1 \star}$, Alex Walmsley ${ }^{2}$ and Victoria Simpson ${ }^{2}$ \\ ${ }^{1}$ Consultant Psychiatrist, Clock View Hospital, Liverpool, Mersey \\ Care NHS Foundation Trust and ${ }^{2} 5$ th year medical undergraduate, \\ University of Liverpool \\ ${ }^{\star}$ Corresponding author.
}

\section{doi: 10.1192/bjo.2021.339}

Objective. This retrospective case series followed emotionally unstable personality disorder (EUPD) patients following initiation of clozapine on an off-licence basis, aiming to examine tolerance by determining side effect prevalence and treatment cessation frequency, as well as examining efficacy, by investigating number of hospital re-admissions and symptom control.

Case report. This case series captured the experiences of 11 EUPD patients under the care of Mersey Care NHS Foundation Trust, all of whom had, at some time in the past five years, been initiated on clozapine. All patients were white British females, with a median age of 31 . The median daily dose of clozapine was $300 \mathrm{mg}$. Most patients had significant psychiatric comorbidities, as well as illicit substance and / or alcohol misuse.

Whilst prescribed clozapine, patients were only admitted to hospital once on average and this was commonly for clozapine re-titration. Whilst in hospital, rates of self-harm were low, but ligaturing and suicide attempts showed higher prevalence. Patients still demonstrated self-harming behaviour out of hospital leading to $\mathrm{A}$ and $\mathrm{E}$ presentations. In the community, contacts with the police were minimal, with only two patients undergoing Section 136 assessments or arrests.

All patients reported side effects from clozapine - usually hypersalivation, over-sedation and constipation. All 11 patients experienced sinus tachycardia. Eight patients temporarily ceased taking clozapine at some point. In three patients, discontinuation of clozapine was as a result of intolerable side effects. Three patients experienced neutropenia, which subsequently resolved. Only two patients had a body mass index within healthy range. Discussion. Despite patients reporting clozapine to provide symptomatic benefit for their EUPD, and improved their engagement with mental health services, prevalence of self-harm and of $\mathrm{A}$ and $\mathrm{E}$ presentations remained high, indicating the importance of community support and concomitant psychotherapeutic treatment. Patients with more robust community support showed greater adherence to clozapine.

High prevalence of side effects and obesity in these patients, in addition to risk of developing neutropenia, highlights the importance of rigorous monitoring after initiating clozapine. It is reassuring that, despite development of neutropenia in some patients, this recovered quickly, and clozapine treatment could resume.

Conclusion. Clozapine may be an effective pharmacological treatment for enabling EUPD patients to engage more therapeutically with services. Clozapine may be of greater benefit to those with more stable, less chaotic lives. Although diminished, patients still show self-harming behaviour and need for A and E admissions and re-hospitalisation. Side effects of clozapine are common and regular monitoring is required.

\section{A haven for an extremely disturbed young person}

\section{Rummana Khan*}

Consultant Child \& Adolescent Psychiatrist, Leicester Partnership NHS Trust

${ }^{*}$ Corresponding author.

doi: 10.1192/bjo.2021.340

Objective. The reason to share this case is to high light the lack of resources in mental health services which can delay the provision of appropriate care and this can have negative impact on child health outcomes.

Background. A 10-year-old boy was referred to CAMHS. He presented with extremely challenging behaviours. After first appointment with CAMHS he attacked his father and nurses. He had to be restrained multiple times. He started to use wooden chair as a weapon, threatened to harm others and threatened to urinate on staff. He tried to kill him-self by ligature. Mental health act assessment was completed and when a decision was reached that detention under the mental health act was appropriate, no appropriate bed was available. He was admitted under Section II of MHA to paediatric ward where he remained for one week (with 2:1 CAMHS support). Then he was transferred to an inpatient CAMHS unit which was commissioned for children over 12 years of age. At a later date mental health tribunal panel upheld the section. After few days he was transferred to an age appropriate in-patient mental health bed. He stayed there for roughly 6 months and was discharged with a diagnosis of ADHD and Autistic Spectrum Disorder. There was a long delay in discharge, until appropriate specialist residential placement could be identified and he was transferred there. He is well settled now in the placement.

Case report. Legal advice was later taken on this case. MHA 1983, Human Rights Act, Children Act 1989, Criminal Law Act 1967 and Code of Practice 2015 were considered and it was agreed that it was appropriate to use MHA 1983. There was discussion whether the Children Act could be relied on instead, but in 